\title{
Timing in the Cerebellum and Cerebellar Disorders
}

\author{
Rebecca M.C. Spencer*
}

1

\section{Introduction}

Over the past century, the function of the cerebellum was largely assumed to be that of movement coordination. This assumption, along with its geographic segregation from the cortex, led the cerebellum to be overlooked in many early neuroimaging studies. Yet the cerebellum contains roughly $10 \%$ of the brain's mass (Hutchinson et al., 2003; Swanson, 1995). Perhaps more striking, the cerebellum contains $65-75 \%$ of the total neurons of the brain (Herculano-Houzel, 2010). In other words, for every one cortical neuron there are three to four cerebellar neurons. Moreover, neurons of the cerebellum are uniquely arranged into arrays of neural loops. These loops repeat millions of times throughout the cerebellum, and are arranged into lobules. Those lobules are, in turn, composed of folia, structures reminiscent of the fractalian structure of a tree or cauliflower (Voogd and Glickstein, 1998). Thus, the cerebellum is architecturally unique and capable of performing a redundant computation. It has been proposed that the computation performed by the cerebellum is that of timing (Braitenberg, 1983; see review in Spencer and Ivry, 2013).

In humans, focal cerebellar damage is rare. While a hemorrhagic stroke to the cerebellum is particularly rare, ischemic stroke, the more common cerebellar stroke, nonetheless accounts for only $1-7 \%$ of all strokes (Bogousslavsky, Van Melle, and Regli, 1988; Macdonell, Kalnins, and Donnan, 1987; Vemmos et al., 2000). Other focal cerebellar lesions, due to tumors and tumor resection, are also uncommon particularly in adults.

Cerebellar atrophy that typically results in widespread cerebellar damage can arise in a number of ways. Genetic degenerative diseases, primarily spinocerebellar ataxia (SCA), that affect the cerebellum are heterogeneous. SCA is a

* Department of Psychology, Neuroscience and Behavior Program, University of Massachusetts, Amherst, USA.

(C) REBECCA M.C. SPENCER, 2015 | DOI 10.1163/9789004230699_010 
group of autosomal dominant neurodegenerative disorders, many of which result in cerebellar degeneration. Prevalence estimates of SCA vary from .3 to 3 per 100,000 (van de Warrenburg et al., 2002). Moreover, of the approximately 30 different SCA subtypes (Schols et al., 2004), only a handful have a primarily cerebellar foci (e.g., SCA 6, 8, 15/16; Durr, 2010; Marelli et al., 2011). Of the degenerative cerebellar disorders, sporadic idiopathic cerebellar atrophy may have the greatest prevalence, estimated to have a prevalence rate of 8.4 per 100,000 (Muzaimi et al., 2004). Sporadic cases of cerebellar agenesis have also been reported (e.g., Glickstein, 1994; Macchi and Bentivoglio, 1977; Nowak, Timmann, and Hermsdorfer, 2007; Velioglu, Kuzeyli, and Ozmenoglu, 1998). Surprisingly, in many of these cases, motor performance is largely normal, suggesting possible compensatory mechanisms (for a review see: Glickstein, 1994; Macchi and Bentivoglio, 1977).

Seminal neurologists, Joseph Babinski and Gordon Holmes, are both credited for the earliest reports of the deficits associated with cerebellar damage in humans (Holmes, 1939, 1917; Trouillas et al., 1997). Babinski and Holmes described core symptoms associated with damage to the cerebellum - such as slurred speech, unsteady gait, and incoordination - and collectively referred to these as 'cerebellar syndrome'. Holmes, studying survivors of gunshot wounds to the back of the head in World War I, came to use the term 'ataxic' to specifically describe the movements of individuals with cerebellar syndrome. Ataxia remains the commonly used term to describe the movements associated with cerebellar disorders. Ataxia is broadly characterized by postural deficits, impairments in upper and lower limb movements, speech impairments, and oculomotor dysfunction (Trouillas et al., 1997). However, the array of symptomatology varies according to localization and extent of damage (Dichgans and Diener, 1984).

There is evidence that the montage of symptoms associated with cerebellar ataxia is due to impaired timing. Most prominently, ataxic movements are uncoordinated. It has been suggested that the lack of coordination between muscles is due to increased time and temporal variability of muscle initiation for individual sub-movements (Day et al., 1998). Upper and lower limb ataxia is also marked by intention tremor, dysmetria, and dysdiadochokinesis on a clinical exam. Unlike the resting tremor that visibly marks Parkinson's disease, intention tremor is not observed at rest; rather, it is most obvious at the endpoints of visually-guided movements. Intention tremor contributes to dysmetria. Dysmetria is the tendency to overshoot or undershoot a target such as the physician's finger. This, too, may be the result of impaired timing, in this case there is a breakdown in the timing between agonist and antagonist muscle contractions composing the movement (Flament and Hore, 1986). Impaired 
production of rapid alternating movements, termed dysdiadochokinesis, has likewise been attributed to impaired timing of sub-movements (Conrad and Brooks, 1974).

Empirical tests of timing in cerebellar patients also support the functional contribution of the cerebellum to movement timing. A repetitive tapping paradigm has been widely used to isolate motor timing in the laboratory setting. In such a task, participants are presented with a metronome that sets the goal pace, for instance a 50oms interval marked by brief metronome tones. At the beginning of a trial, the participant's goal is to tap at this goal pace (synchronization phase). After the metronome disengages (typically following $5^{-10}$ paced intervals), the participant's goal is to continue moving at that pace until the trial's end (continuation phase; e.g., after $15^{-20}$ unpaced taps). Importantly, individuals with cerebellar damage are impaired in repetitive tapping with a range of effectors (Ivry, Keele, and Diener, 1988). Specifically, temporal variability is greater for those with cerebellar damage relative to control groups (typically matched in age, education, handedness, and gender).

Moreover, a corpus of studies suggests that motor timing deficits in cerebellar patients may underlie movement inaccuracies. In a study of overarm ball throwing, inaccuracies of throws produced by individuals with cerebellar lesions were related to increased variability in the timing of the ball release within the rotation of the arm (Hore, Timmann, and Watts, 2002; Timmann, Richter, Bestmann, Kalveram, and Konczak, 2000; Timmann, Watts, and Hore, 1999). Likewise, ataxic speech may be greatly accounted for by a deficit in motor timing. The duration of syllable repetitions is slowed and irregular in ataxic speech. Speech slowing may be similar to that observed in individuals with Parkinson's disease (Ackermann et al., 1997b; Canter, 1963). However, cerebellar lesions also yield and increase the temporal variability of articulatory timing (Ackermann and Hertrich, 2000; Schalling, Hammarberg, and Hartelius, 2007).

These motor timing deficits are consistent with historical views of the cerebellum as being a motor structure, particularly for movement coordination. However, cerebellar patients are also impaired in non-motor, temporal perception tasks. A duration discrimination task provides an ideal probe of temporal perception with little motor demands. In a duration discrimination task, participants are presented with two intervals and asked to judge whether the second is shorter or longer than the first. By parametrically varying the difference between the intervals, a discrimination threshold and variability in discrimination performance can be obtained. While thresholds are generally similar between cerebellar patients and matched controls, variability in performance is greater for those with cerebellar lesions (Ivry and Keele, 1989) suggesting that the internal clock is 'noisy' (Ivry and Spencer, 2004a). Notably, differences 
are only observed for temporal discriminations. When the task requires discrimination of the amplitude (or loudness) of the tones (Ivry and Keele, 1989) or frequency (Casini and Ivry, 1999), performance does not differ between individuals with cerebellar lesions and controls.

The impairment in temporal perception may also underlie the deficit in speech perception observed in individuals with cerebellar ataxia. Distinguishing temporal phonetic features is critical to discriminating speech successfully. In English, to distinguish between 'rabid' and 'rapid' relies on precise temporal coding. Using similar words from the German language, such as 'boden' and 'boten', Ackermann and colleagues (1997a) found impaired discrimination of speech sounds in individuals with bilateral cerebellar degeneration, and this deficit was specifically associated with the temporal cues innate in the speech stimuli.

A set of recent studies further illustrates that cerebellar lesions also lead to impaired perceptual timing of interception, a skill necessary to catch a ball or to catch a closing elevator. In one study, individuals with cerebellar ataxias and matched control participants played a computer game in which a moving target must be intercepted by a 'cannon ball'. As such, the task required integration of an estimate of the speed of the moving target as well as the speed of the cannon ball once it was released (through a finger press). Participants with cerebellar ataxia performed significantly worse at the task than healthy controls (Bares et al., 2007; Bares et al., 2010a,b) and participants with Parkinson's disease (Bares et al., 2010a) supporting a role of the cerebellum in this form of perceptual time estimation.

In addition to temporal production and perception tasks, the cerebellum contributes uniquely to temporal aspects of associative learning. While early studies focused on the contribution of hippocampal and cortical areas (Gormezano et al., 1962; Oakley and Russell, 1972), only cerebellar lesions eliminate learning of the conditioned response (McCormick and Thompson, 1984). This is often demonstrated with an eye blink classical conditioning paradigm. In a typical eye blink conditioning task, the individual experiences trials in which an air puff to the eye, which causes an unconditioned eye blink, is paired with a tone. Over time, the individual is conditioned to respond to the tone alone with an eye blink. When a delay is implemented between the tone and the air puff, precise timing of the eye blink in response to the tone is necessary in order for the response to be adaptive (i.e., eye closed at the time at which an air puff might arrive). In animal and human cerebellar lesion studies of eye blink conditioning, the subjects are able to learn the conditioned response following a cerebellar lesion. Importantly, however, the adaptive timing of the response is lacking (Gerwig et al., 2003; Perrett, Ruiz, and Mauk, 1993; 
Woodruff-Pak, Papka, and Ivry, 1996). In other words, the cerebellar lesioned animal will appropriately learn the response (a blink), but this response is illtimed relative to the conditioned stimulus and, thus, not adaptive.

Collectively, these data demonstrate that temporal impairments may underlie ataxic movements. Perhaps more importantly, cerebellar lesions result in impaired timing in tasks explicitly measuring temporal perception and production (for reviews see Ivry and Spencer, 2004b; Ivry, 1996).

While cerebellar lesions result in timing impairment, this alone does not necessarily implicate the cerebellum in functioning as a single neural clock (i.e., a specialized dedicated timekeeper). Lesions in other subcortical or cortical regions may yield similar timing deficits that would support timing as a distributed function incorporating a network of brain areas (i.e., a distributed dedicated timekeeper). A third alternative is that multiple brain areas may have the ability to time depending on the relevant modality (i.e., intrinsic timing; for a review see Ivry and Schlerf, 2008).

A wealth of individual difference studies using repetitive tapping tasks, collectively favor a neural clock that functions across a range of durations. This individual difference work is carried out under the assumption that if there is a single neural clock, then a person with a "good clock" should perform well across a range of timing tasks and, likewise, a person with a rather "poor clock" should perform relatively poorly across tasks. In other words, one should see highly significant correlations in temporal performance across timed tasks. Indeed, temporal variability for a repetitive finger tapping task significantly correlates with variability for tapping with a range of other effectors. For instance, finger tapping (flexion/extension at the metacarpophalangeal joint) at a rate of 40oms per tap correlates with tapping with the arm (flexion/extension of the glenohumeral-shoulder-joint) and tapping the jaw (excursions of the temporomandibular joint) at the same rate (Franz, Zelaznik, and Smith, 1992). Likewise, temporal variability in the repetitive finger tapping task correlates with temporal variability for a repetitive foot tapping task (flexion/ extension at the talocrural-ankle-joint; Keele et al., 1985).

Moreover, individual difference studies suggest that the capacity of the neural clock is not limited to motor tasks. Performance on repetitive tapping tasks is also predictive of performance on non-motor timing tasks (Keele et al., 1985). The duration discrimination task (described above) has been used to demonstrate this point. Importantly, performance on repetitive tapping tasks is 
predictive of performance on the duration discrimination task, suggesting a shared timing process across motor and perceptual timing tasks.

Thus, there is certainly reason to believe that temporal performance draws on a single neural resource. Taken in conjunction with the evidence provided in Section 2, evidence points to the cerebellum as being this neural clock. While this proposed function of the cerebellum as a timekeeper has gained favor, recent work in our lab and others has focused on limitations of the cerebellar clock.

\section{4}

\section{Limitations on Cerebellar Timing}

\subsection{Event versus Emergent Timing}

Given the rather consistent pattern of correlations that our lab and others have observed amongst timing tasks (Franz, Zelaznik, and Smith, 1992; Keele and Hawkins, 1982; Keele et al., 1985), we were surprised to find a lack of correlation between temporal variability on a repetitive finger tapping task and a repetitive continuous circle drawing task. As an aside, continuous cycling (either circling or 'wagging' of an effector) was of interest given that research on motor coordination, another hypothesized function of the cerebellum, was largely based on continuous bimanual movements (e.g., Carson et al., 1997; Haken, Kelso, and Bunz, 1985; Kelso, 1984; Semjen, Summers, and Cattaert, 1995). Timing of continuous movements is also particularly unique as temporal variability is remarkably low for healthy young adults. While the coefficient of variation, a measure of variability normalized to movement time, is typically $5^{-8 \%}$ of the mean movement time for finger tapping (Keele et al., 1985; Theoret, Haque, and Pascual-Leone, 2001), it is as low as $3-5 \%$ for circle drawing (Spencer et al., 2003; Spencer and Zelaznik, 2003; Zelaznik, Spencer, and Ivry, 2002). Most importantly, these measures of temporal variability do not correlate across these two tasks (Robertson et al., 1999; Zelaznik, Spencer, and Ivry, 2002).

In a continuous circle drawing task, participants are given a circle template to equate movement amplitude across participants. A visual target at the top of the circle template assists participants in aligning movements to the metronome; participants are to pass through this target coincident with the metronome beep during the paced portion of a trial, just as they are to touch the table coincident with the metronome for the repetitive tapping task (Robertson et al., 1999; Zelaznik, Spencer, and Ivry, 2002). Nonetheless, when one performs this task, it is quite obvious that this movement goal is much less salient for the continuous circle drawing task than it is for the repetitive finger tapping task. 
This led us to propose the Event Timing Hypothesis: Movements or intervals that are marked by salient features would share a timing process, while movements lacking such a structure would not be amenable to such a process (Ivry et al., 2002). To test this hypothesis, we compared variability on the continuous circle drawing task to a discrete circle drawing task. In the discrete circle drawing task, participants traced the circle template for every other movement interval and paused at the top of the trajectory for the intervening intervals. Thus, these tasks were equated for movement speed, amplitude, and biomechanical demands. In spite of this, there were no correlations between continuous and discrete circle drawing. Consistent with the Event Timing Hypothesis, however, performance on the discrete circle drawing did predict performance on the repetitive tapping task (Zelaznik, Spencer, and Ivry, 2002).

At this point, we know little about how continuous movements are timed. It has been suggested that timing for such tasks may be emergent. The idea of emergent timing is derived from Dynamical Systems Theory applied to studies of motor control (Turvey, 1977). Accordingly, timing for continuous tasks may emerge from the dynamics of the limbs. Alternatively, other properties of the movement, rather than timing being under the direct control of a neural clock, may be under direct neural control and result in well-timed movements. For instance, in a continuous circle drawing task, neural processes controlling movement velocity or jerk (Hogan and Flash, 1987) may result in precise temporal consistency without the direct control of time. Supporting the concept of an array of unique emergent processes, we found that temporal processes underlying continuous circling and continuous line drawing are distinct, and these processes are also independent of that used for discrete movements such as finger tapping (Spencer and Zelaznik, 2003). In other words, there seems to be no single 'continuous movement timer'. To illustrate this, we applied a slope analysis to the variability of timing across these tasks. The slope analysis, the examination of the relation between the timed interval and timing variance, assumes two independent sources of variability to movement timing: A duration-independent source associated with variability in implementing a timed movement command and a duration-dependent source associated with the central clock-like process (Wing and Kristofferson, 1973). When movement time is plotted against temporal variability for a given task a linear relationship is observed, particularly for millisecond to second range tasks (Gibbon, 1977; Gibbon and Church, 1990). Importantly, if two tasks share a neural timing process, they should have the same variability-interval relationship, or shared slope (Ivry and Corcos, 1993; Ivry and Hazeltine, 1995). Consistent with this, we observed the same 
variability-interval slopes for two line drawing tasks. Likewise, the slope was the same for two continuous circle drawing tasks. However the slopes were unique for continuous circle drawing relative to continuous line drawing. Thus, these results suggest a degree of independence for timing processes underlying continuous movement timing, but within any given task (e.g., variants of a continuous circling task), a shared timing source is used (Spencer and Zelaznik, 2003).

Even under indirect control, however, it is speculated that continuous movements may rely on a neural 'clock' to align early movement cycles to the metronome-instructed temporal goal. According to this Transformation Hypothesis, early cycles of a repetitive movement may utilize an explicit timing process to set the pace of the movement before a non-clock process (e.g., velocitybased control) may take over. Supporting this, Zelaznik and colleagues (2005) again compared temporal performance for repetitive finger tapping and repetitive continuous circle drawing but focused analyses on the initial, early cycles of the trials. Variability in the first cycle of these tasks was significantly correlated. Yet, consistent with work reviewed above, temporal variability for subsequent cycles did not correlate. This suggests that tasks with a precise temporal goal may initially require the event-based temporal representation, or internal clock, even though other non-clock timing processes may take over once the appropriate rate is adopted consistent with the Transformation Hypothesis (Zelaznik et al., 2005).

Individual difference studies into event timing in healthy individuals have provided insight as to how the cerebellar 'clock' measures time for movements and percepts. That is, the cerebellum may serve as a timer when salient features are available by which to start and stop the neural clock (Ivry et al., 2002). If such were the case, cerebellar lesions would yield impaired timing on repetitive movement tasks and duration perception tasks that require the timing between discrete events (e.g., metronome beeps, table touches when finger tapping), deficits that have been consistently reported. However, if the cerebellum provides only discrete/eventful timing measures, this yields a rather counterintuitive prediction, that is that individuals with cerebellar lesions may be unimpaired in the timing of continuous movements. We tested this in a series of studies of individuals with cerebellar ataxia.

In one such study, we compared temporal performance on repetitive finger tapping (on a table top; i.e., 'eventful'), continuous circle drawing, and discrete circle drawing (Spencer et al., 2003). The goal movement time for all tasks was $800 \mathrm{~ms}$ per cycle. Participants were individuals with focal, unilateral lesions of the cerebellum due to stroke or tumor resection. With focal 
unilateral cerebellar lesions, impairments are observed unilaterally, in the ipsilesional limb (the cerebellum receives crossed projections from the cortex, and therefore, control is ipsi- rather than contra-lateral; Franz, Ivry, and Helmuth, 1996; Ivry, Keele, and Diener, 1988). Thus, this group allows for within-subject comparisons of the limb under control of the lesioned cerebellum to the unimpaired limb. Similar to previous studies (e.g., Ivry, Keele, and Diener, 1988), we found that temporal variability was greater with the ipsilesional, impaired limb relative to the contralesional, unimpaired limb for the table tapping task. Likewise, variability was greater for the ipsilesional limb relative to the contralesional limb for the discrete circle drawing task. Importantly, consistent with the predicted role of the cerebellum for timing of events, there was no difference in performance across limbs for the continuous movement task. Strikingly, the coefficient of variation was remarkably low, only about $3 \%$ for both the unimpaired and impaired limb in the cerebellar lesion group for the continuous circle drawing task (Figure 9.1; Spencer et al., 2003).

In a second experiment, we compared temporal variability for the repetitive finger tapping task under four conditions: (1) tapping on a table top, a variant of the task most commonly used in previous studies, at a rate of 10ooms per cycle; (2) a discrete air tapping task, also at a rate of $1000 \mathrm{~ms}$ per cycle, in which taps were made with the finger held away from any table surface and movements were instructed to be 'discrete and staccato-like'; (3) a continuous air tapping task, at a rate of $1000 \mathrm{~ms}$ per cycle, in which taps were, again, made without surface contact and participants were instructed to "move smooth and

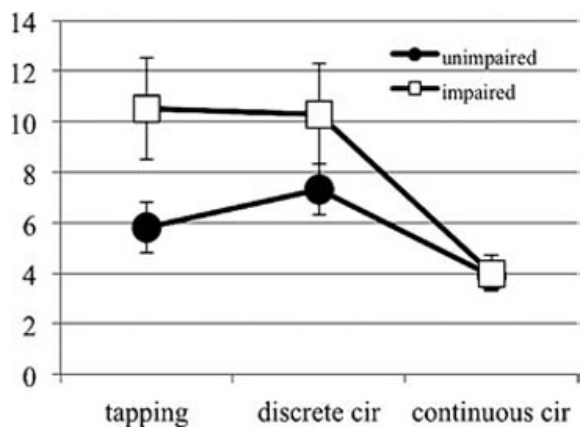

FIGURE 9.1 Coefficient of variation (standard deviation divided by mean movement time) for repetitive finger tapping, discrete circle drawing and continuous circle drawing. Movements were performed by individuals with unilateral cerebellar lesions with both their impaired and unimpaired limbs. Error bars represent standard error.

ADAPTED FROM SPENCER ET AL., 2003. 
continuously"; and (4) a continuous air tapping task at a rate of $500 \mathrm{~ms}$ per cycle (Spencer et al., 2003). Note that the last task, the fast continuous task, was included to equate for the faster velocity of the movement component of each cycle in the discrete tasks relative to the continuous tasks. That is, when tapping discretely, the velocity of the finger must be faster than in the continuous task in order to include a brief pause within the trajectory, thereby making the movement discrete. As such, one might argue that impairments associated with cerebellar lesions in timing of discrete movements, such as table tapping, are simply due to the speed of the movement, a deficit that may not be surprising to observe in conjunction with any movement disorder. Importantly, results were consistent with the Event Timing Theory and the hypothesized role of the cerebellum in timing movements with salient events. That is, for individuals with focal cerebellar lesions, variability was greater with the ipsilesional, impaired limb relative to the contralesional, unimpaired limb for the discrete tasks, table tapping, and discrete air tapping. There was no difference in performance across limbs for either the fast (500ms/cycle) or slow (1000ms/ cycle) continuous air tapping tasks.

We found a similar pattern of results when comparing a group of individuals with bilateral cerebellar lesions (degeneration due to SCA or sporadic cerebellar ataxia) to healthy matched controls. Here, performance with the dominant hand was compared for individuals with bilateral cerebellar ataxia (SCA3, SCA6, or sporadic cerebellar ataxia) and a group of healthy age- and education-matched control participants. Individuals with bilateral cerebellar lesions exhibited greater variability than the control group for the discrete tasks. There was no difference in performance across groups for the continuous movement tasks (Spencer et al., 2003).

Finally, neuroimaging evidence supports the Event Timing Theory of cerebellar function. We examined the Blood-oxygen-level dependent (BOLD) response (i.e., neural activation) using functional magnetic resonance imaging (fMRI) when healthy young adults performed the continuous and discrete air tapping tasks described above. While activation in lobule vi of the cerebellum was observed for both conditions, additional activity (located in the superior vermis) was selectively activated for the discrete movement. This is consistent with the hypothesis that the cerebellum plays a unique role in discrete movement timing (Spencer et al., 2007).

Together, this work demonstrates that the cerebellar clock function is restricted. Specifically, the manner in which the cerebellum computes time, by representing intervals demarcated by salient events, by nature restricts this role to a subset of movement and perceptual tasks that are amenable to such a timekeeper. 


\subsection{Interval Range of the Cerebellar Timing: Long versus Short Interval Timing}

Importantly, tasks discussed to this point involved intervals in the millisecond range, mostly less than 200oms. For intervals greater than this, the contribution of the cerebellum to timing may decrease, with increasing weight of the non-temporal processes contributing greatest to performance variability. For instance, Mangels, Ivry, and Shimizu (1998) compared performance on a short, 40oms, interval task with performance on a long, $4 \mathrm{~s}$, interval task in individuals with cerebellar lesions and age- and education- matched healthy control participants. The task was a duration perception task, ruling out the contribution of motor deficits to short versus fast movements. They found that individuals with cerebellar lesions were impaired, relative to healthy control participants, in perception of both the short and long intervals. To examine whether the neocortex further supports this timing function, via attention or working memory mechanisms, a group of individuals with focal lesions to the prefrontal cortex were also included. Individuals with lesions of the prefrontal cortex were impaired, relative to healthy controls, on the long interval perception tasks. There was no difference in performance for these two groups on the short, 40oms, perception task. The authors suggest that, for longer intervals, cortical contributions are necessary to maintain the standard in working memory and to attend to the task over the long interval. However, it may be somewhat surprising that the deficit in performance was not reduced for the longer, $4 \mathrm{~s}$, interval for those with cerebellar lesions. One explanation for this finding is that, for longer interval timing, a sub-interval timing strategy may be used. That is, when faced with the task of timing a 4-s interval, one may internally time four 1-s intervals. As such, the cerebellar contribution to millisecond timing would be evident in this way.

In a meticulous analysis of temporal perception and production across a range of suprasecond intervals (2-12 s), a recent study found further evidence that the cerebellar contribution is greatest for short intervals although the deficit is still present for longer intervals (Gooch et al., 2010). In the duration production task under consideration, participants were required to reproduce the duration of a 2, 4, 6, 8, 10 or $12 \mathrm{~s}$ stimulus (a red square on a computer screen). The authors report a significant group (cerebellar patients versus controls) by interval interaction, reflecting highly variable temporal performance for the cerebellar patients relative to controls at the shortest intervals $(2 \mathrm{~s}$ and, less so, $4 \mathrm{~s}$ ) with little group differences observed at the longer intervals. Likewise, a temporal estimation task, requiring participants to produce intervals of 2, 4, 5 , 6,10 or $12 \mathrm{~s}$ from memory, revealed consistently worse performance for individuals with cerebellar lesions compared to controls across all intervals, 
although the deficit was again greatest at the shorter durations. Notably, when the patient group was divided into those with middle-to-superior cerebellar lesions and those with damage outside of middle-to-superior cerebellum, only those with middle-to-superior damage differed from controls. This suggests that the cerebellar clock may be localized to superior cerebellum, an issue we will return to below.

Individuals with Parkinson's disease are also reported to be impaired in temporal perception and production tasks. Movements of individuals with Parkinson's disease are visibly slow, a deficit termed bradykinesia. As such, one might predict that individuals with Parkinson's disease would be slow on a repetitive tapping task. Counter-intuitively, however, these individuals actually tend to speed up movements (Harrington et al., 2004; Ivry and Keele, 1989; O'Boyle, Freeman, and Cody, 1996). Whether temporal variability is increased in individuals with Parkinson's disease, as would be expected for an impaired neural clock, is unclear. Considering intervals in the millisecond range (less than 2 s), some studies report increased temporal variability in Parkinson's disease (Harrington, Haaland, and Hermanowicz, 1998) relative to matched controls, while others find no difference (Ivry and Keele, 1989; Spencer and Ivry, 2005). While Parkinson's disease patients are often examined as a model of basal ganglia dysfunction, isolation of the disease to basal ganglia is not pure. Importantly, Aparicio and colleagues (2005) found that individuals with focal lesions of the basal ganglia, most in the putamen, some extending to the caudate, internal capsule or globus pallidus, due to stroke, were unimpaired on a repetitive tapping task. Variability of taps, with a goal pace of 40oms, did not differ for basal ganglia lesion patients relative to healthy controls nor was there a difference in variability between the contralesional, impaired limb and the ipsilesional, unimpaired limb. While this work remains to be replicated, it calls into question the conclusion of some that the basal ganglia is an internal clock for millisecond range intervals.

It is worth considering that the basal ganglia may contribute to timing of suprasecond intervals, those intervals greater than the 200-200oms that seems to be the expertise of the cerebellum. Supporting this, Matell and colleagues (2003) illustrated the ability of the striatum to time intervals of 10 and $40 \mathrm{~s}$. By recording striatal neurons in rats trained to time press a lever 10 or $40 \mathrm{~s}$ after a stimulus onset, it was clear that these neurons distinguished between the two intervals just as response rates were distinct for the two trial types. More importantly, a relationship between the time of peak firing and performance was observed (Matell, Meck, and Nicolelis, 2003).

The selective role of basal ganglia for suprasecond intervals and not subsecond intervals was distinguished in a recent study in humans using deep brain 
stimulation (DBS) to the subthalamic nucleus (STN; Wojtecki et al., 2011). DBS is used to treat symptoms of Parkinson's disease. When the stimulation was off, these participants were impaired relative to matched controls; specifically, $15^{-\mathrm{s}}$ intervals were underestimated in length. Low frequency $(10 \mathrm{~Hz})$ stimulation resulted in further underestimation when reproducing the 15 -s interval. Conversely, high frequency $(130 \mathrm{~Hz})$ stimulation reduced the amount of underestimation, resulting in performance similar to that of controls. However, neither high nor low frequency DBS to the same region influenced duration discrimination in the millisecond range (800-16ooms), supporting a distinction in the temporal range that the basal ganglia may expertly time.

The corollary to the consideration of a separate, perhaps basal gangliabased, timing process for longer intervals, is to consider whether there is a lower limit to the cerebellar timing function. Can the cerebellum time intervals on the range of $5^{\circ}$ or 10oms? Mounting evidence suggests that timing for such ultra-short intervals is also distinct. In fact, ultra-short intervals may be unique from other intervals in that timing for ultra-short intervals might not draw on the function of a single neural clock but rather the timing process may be modality dependent, drawing on cortical areas associated with that modality. Termed the State-Dependent Network model, Karmarkar and Bounomano (2007) proposed that networks of neurons within a given cortical region can accurately detect time. The basis for the State-Dependent Network model is that time is implicitly represented in the synaptic properties or state of a neural network (Buonomano and Mauk, 1994; Karmarkar and Buonomano, 2007; Yamazaki and Tanaka, 2005). By simulating a series of state-dependent networks, Karmarkar and Buonomano (2007) showed that, when presented with a brief (e.g., 10oms) interval marked by two tones, the first tone of the pair will generate activity in the network. This activity then changes predictably over time and includes fast and slow inhibitory post-synaptic potentials and shortterm synaptic plasticity in the connections between nodes in the network. When the second tone of the interval arrives, the dynamical state of the network has been changed as a result of the first tone, so there is a different pattern of activity in the network in response to the second tone even if the second tone is identical to the first tone in duration, pitch, and frequency. The temporal interval defined by the two tones can be interpreted from the final state of the network. Importantly, if the stimulus (10oms interval marked by two tones) is preceded by any event, the state of the network is changed. As such, if standard and test intervals are presented in two different contexts, the dynamics of the state-dependent network would differ and the ability to encode duration would fail. This creates a 'reset problem', which has been used to demonstrate support for the State-Dependent Network (SDN) hypothesis. In the Reset Task 
designed to test this problem, similar to the duration perception task described above, human participants were presented with an interval of approximately 10oms marked by two tones and were to respond as to whether the interval was short or long relative to a 10oms standard interval (Figure 9.2A; Spencer, Karmarkar and Ivry, 2009). Performance was similar if the test interval was preceded by a distractor interval also of 100ms. This result is consistent with the SDN hypothesis given that a fixed distractor interval would allow for building a stable representation of this fixed state based on trial-by-trial feedback. More importantly, when the distractor interval was of varying length (50-150ms), discrimination thresholds were greatly increased relative to the no-distractor condition. This is consistent with the prediction of the SDN hypothesis. That is, that the variable length distractor results in the network being in a different state each time the target interval is presented and a stable representation cannot be extracted (Karmarkar and Buonomano, 2007).

Recently, we revisited this effect, considering attentional issues that may also produce the context effects observed in the Reset Task (Spencer, Karmarkar, and Ivry, 2009). We predicted that using very brief intervals (i.e., 10oms and 100ms $+/-50 \mathrm{~ms})$, which may trigger automatic attentional capture (Posner, 1978), imposed unique sources of variability. In other words, attention would be drawn to the distractor interval by attentional capture thereby impairing attention when presented with the target interval. To test this, we replicated the Reset Task using both conditions from the original Karmarkar and Buonomano study (2007; approximately 10oms for both the distractor and target intervals; 100-100 task) and a novel condition with a 300ms distractor and 100ms target interval (300-100 task). First, with respect to the 100-100 task, our results replicated those of Karmarkar and Buonomano (2007) in that thresholds were elevated in both the fixed and variable distractor conditions, relative to the no-distractor conditions, and thresholds were greatly increased in the presence of a variable distractor interval (Figure 9.2B; Spencer, Karmarkar and Ivry, 2009). This was not the case in the 300-100 task. While there was a small but significant main effect of a distractor, performance with a variable distractor was not worse than a fixed distractor in this condition. This result limits the range of the SDN model. That is, the original model assumes that any distractor interval (100- or 300-ms) should alter the state of the network when presented with the target interval. Rather, these results are consistent with our hypothesis that unpredictable ultra-short intervals $(<150 \mathrm{~ms})$ may disrupt timing of the target interval. Importantly for the purposes of the present discussion, this work supports a distinction in timing for ultra-short (50-150ms) and longer millisecond range (200-200oms) timing. Furthermore, we report that variants of the Reset Task with a 30oms target interval and either a 100ms (100-300 task) or $300 \mathrm{~ms}$ 
(300-30o task) distractor interval also failed to find the increased threshold in the variable distractor condition relative to the fixed condition or no-distractor conditions. These results indicate how attentional processes can influence the perception of brief intervals, as well as point to important constraints of SDN models. As such, we posit that the SDN model, which proposes that timing may be computed in cortical regions supporting the task modality, may support ultra-short interval timing (<200ms) but not millisecond range timing (200200oms; Spencer, Karmarkar, and Ivry, 2009).

5

\section{Localization of Timing within the Cerebellum}

Given the evidence supporting the function of the cerebellum in millisecond range timing, we turn to consideration of where such a function might be located within the cerebellum. Architecturally, the cerebellum is composed

A)

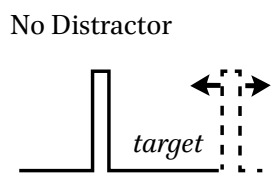

Fixed Distractor
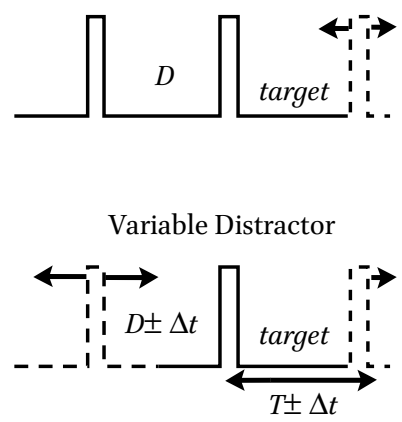

short or long?
B)
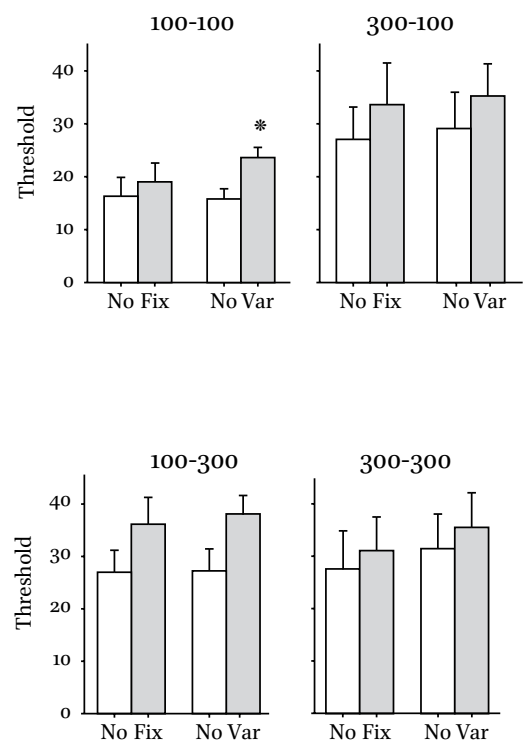

FIGURE 9.2 A) The Reset Task described by Karmarkar and Buonomano (2007). Participants judged the length of the target $(T)$ relative to a previously presented standard interval when the target was presented without a distractor (top), with a fixed interval distractor ( $D$; middle), or a variable length distractor (bottom). B) Consistent with Karmarkar and Buonomano (2007), we found an increased threshold with a variable distractor condition for the 100-100 condition. This was not the case in the 100-300, 300-300, or 300-100 conditions.

ADAPTED FROM SPENCER, KARMARKAR, AND IVRY, 2009. 
of repeating loops. These loops underlie three longitudinal divisions - the vermis, the paravermis, and the hemispheres. Each of these divisions is, in turn, composed of multiple lobules. This elaborate arrangement may either suggest a highly specialized single map (Apps and Hawkes, 2009) or multiple functional units (Grodd et al., 2001). Mounting evidence suggests a specialized role of superior cerebellum in timing. As described above, timing impairments are exaggerated in individuals with superior cerebellar lesions relative to those with inferior lesions (Gooch et al., 2010). Like Gooch and colleagues (2010), Harrington and colleagues (2004) studied timing in individuals with superior versus inferior cerebellar lesions and found that timing impairments were associated with superior lesions. In this study, Harrington and colleagues compared two groups of cerebellar lesion patients: those with lesions to inferior cerebellum (approximately lobules VII-X) and those with superior cerebellar lesions (approximately lobules I-VI). Those individuals with superior, but not inferior cerebellar lesions, were more variable relative to healthy controls on the duration perception task with standards of 300 and 6ooms, although this reached only trend-level for significance $(p=.07$; see Ivry and Spencer, 2004a). Moreover, those with superior cerebellar lesions were more variable than the control group on a repetitive finger tapping task at both 300 and 6ooms. Specifically, total variability was modeled to dissociate the variability due to the central timekeeping process (i.e., 'clock' variability) from variability due to noisy motor implementation (i.e., motor variability; Wing and Kristofferson, 1973). The deficit observed in individuals with superior cerebellar damage was associated with the former, supporting an impaired clock process in superior cerebellum.

Functional imaging studies, by and large, find anterior cerebellum is active in conjunction with timed movement and perception tasks. For instance, using positron emission tomography (PET), Penhune and colleagues (1998) found that performance on the repetitive finger tapping task produces activation in lobule $\mathrm{V}$ beyond that seen in a control condition in which participants simply listened to the metronome. Likewise, neuroimaging of perceptual timing tasks also yields cerebellar activation. However, the localization within the cerebellum for these tasks is less clear. For example, one study of visual timing pointed to a critical region around the border of left Crus I/II, activation (Lewis and Miall, 2003). Other studies have pointed to a region within lobule vi (Aso et al., 2010) or superior vermis (Tesche and Karhu, 200o; Xu et al., 2006).

The anterior cerebellum has a known somatotopic arrangement, akin to the homunculus of the motor cortex. Using functional neuroimaging, mapping of the activation in this region suggests an inverted body map with the foot superior to the hand and the hand superior to the lips and facial representations 
(Grodd et al., 2001; Habas, Axelrad, and Cabanis, 2004; Nitschke et al., 1996; Rijntjes et al., 1999). It has been proposed that underlying this body map is a series of interval representations (Ivry, 1996). In other words, the cerebellum may have an effector by interval arrangement akin to the ocular dominance columns in visual cortex. This model would be consistent with the observed unilateral impairment associated with lateralized cerebellar lesions.

In addition to the somatotopic organization of the anterior lobe, somatotopy has been observed in the inferior aspect of the posterior lobe of the cerebellum (lobules VIIB and VIII; Habas, Axelrad, and Cabanis, 2004; Rijntjes et al., 1999) and, most recently, we have reported evidence of a somatotopic arrangement in lateral cerebellum, lobules VI and VII (Schlerf, Verstynen, Ivry, and Spencer, 2010). In that study, we used fMRI to map movements of the fingers and toes that were either simple (i.e., flex/extend all fingers/toes simultaneously) or complex (i.e., flex/extend individual digits sequentially). Movements were repetitive at a rate of approximately 500ms. For all movements, ispilateral activation was observed in anterior cerebellum and this activation was somatotopically organized, that is, foot movement responses were distinct from, and inferior to, hand movement responses. Interestingly, when movements were complex, activation was also observed in lobules VI and vir. This lateral cerebellar activation was particularly noteworthy for two reasons. First, unlike the unilateral activity observed in the anterior cerebellum, activation in lateral cerebellum in conjunction with complex movements was bilateral. Second, the activation was somatotopically arranged here as well. This is particularly interesting in lateral cerebellum given that this region has previously been associated with the non-motor functions of the cerebellum (Akshoomoff and Courchesne, 1992; Desmond, Chen, and Shieh, 2005). Such a role is supported by connectivity studies, which illustrate that lateral cerebellum is functionally connected to prefrontal cortex (Kelly and Strick, 2003; Krienen and Buckner, 2009; Middleton and Strick, 2001). Thus, finding somatotopic organization here would be akin to finding somatotopic organization in the frontal cortex. To make sense of this arrangement, from these observations, we propose that the lateral cerebellum may support the anterior cerebellar function under conditions with high cognitive demands - such as movements with high complexity (Schlerf, Verstynen, Ivry, and Spencer, 2010) or working memory when a task requires response mapping (Spencer and Ivry, 2009).

It should be clear from this Chapter that the cerebellum plays a critical role in timing and, for this reason, timing deficits are central to the impairments 
associated with cerebellar lesions and disorders. However, this chapter also emphasizes a critical distinction in temporal processing for ultra-short intervals, millisecond, and supra-second intervals. Timing of ultra-short intervals (approximately those less than 20oms) may utilize SDN in relevant cortical regions. Supra-second intervals (approximately those greater than $2 \mathrm{~s}$ ) may utilize basal ganglia processes with an increase in weight from cortical areas, such as the frontal cortex, for cognitive demands such as working memory. Central to our review is the cerebellum's role for millisecond range intervals (approximately 200-2000ms). While such a distinction may seemingly diminish the functional capacity of the cerebellum, we posit that a deficit in millisecond range timing has substantial ramifications as evidenced by the ataxic movements and impaired perceptions of individuals with cerebellar lesions and disorders.

\section{References}

Ackermann, Hermann et al. 1997a. "Categorical Speech Perception in Cerebellar Disorders" Brain and Language 60: 323-31.

Ackermann, Hermann et al. 1997b. "Kinematic Analysis of Articulatory Movements in Central Motor Disorders" Movement Disorders 12(6): 1019-27.

Ackermann, Hermann, and Ingo Hertrich. 2000. "The Contribution of the Cerebellum to Speech Processing” Journal of Neurolinguistics 13: 95-116.

Akshoomoff, Natacha A., and Eric Courchesne. 1992. "A New Role for the Cerebellum in Cognitive Operations" Behavioral Neuroscience 106: 731-8.

Aparicio, Paul D., Joern Diedrichsen, and Richard B. Ivry. 2005. "Effects of Focal Basal Ganglia Lesions on Timing and Force Control" Brain and Cognition 58: 62-74.

Apps, Richard, and Richard Hawkes. 2009. "Cerebellar Cortical Organization: A OneMap Hypothesis" Nature Reviews Neuroscience 10(9): 670-81.

Aso, Kenji et al. 2010. "Cerebro-Cerebellar Interactions Underlying Temporal Information Processing" Journal of Cognitive Neuroscience 22(12): 2913-25.

Bares, Martin et al. 2007. "Impaired Predictive Motor Timing in Patients with Cerebellar Disorders" Experimental Brain Research 180(2): 355-65.

Bares, Martin et al. 2010a. "Predictive Motor Timing Performance Dissociates between Early Diseases of the Cerebellum and Parkinson's Disease" Cerebellum 9(1): 124-35. Bares, Martin et al. 2010b. "The Neural Substrate of Predictive Motor Timing in Spinocerebellar Ataxia" Cerebellum 9(1): 124-35.

Bogousslavsky, Julien, Guy Van Melle, and Franco Regli. 1988. "The Lausanne Stroke Registry: Analysis of 1,00o Consecutive Patients with First Stroke" Stroke 19(9): $1083-92$. 
Braitenberg, Valentino. 1983. "The Cerebellum Revisited" Journal of Theoretical Neurobiology 2: 237-41.

Buonomano, Dean V., and Michael D. Mauk. 1994. "Temporal Discrimination and the Timing of Motor Responses" Neural Computation 6(1):38-55.

Canter, Gerald. 1963. "Speech Characteristics of Patients with Parkinson's Disease: Intensity, Pitch and Duration" Journal of Speech and Hearing Disorders 28(3): 221-9. Carson, Richard G. et al. 1997. "The Dynamics of Bimanual Circle Drawing” Quarterly Journal of Experimental Psycholology Section A: Human Experimental Psychology 50(3): 664-83.

Casini, Laurence, and Richard B. Ivry. 1999. "Effects of Divided Attention on Temporal Processing in Patients with Lesions of the Cerebellum or Frontal Lobe" Neuropsychology 13(1): 10-21.

Conrad, B., and V.B. Brooks. 1974. "Effect of Dentate Cooling on Rapid Alternating Arm Movements" Journal of Neurophysiology 37: 792-804.

Day, B.L. et al. 1998. "Influence of Vision on Upper Limb Reaching Movements in Patients with Cerebellar Ataxia” Brain 121(2): 357-72.

Desmond, John E., S.H. Annabel Chen, and Perry B. Shieh. 2005. "CerebellarTranscranial Magnetic Stimulation Impairs Verbal Working Memory” Annals of Neurology 58(4): 553-6o.

Dichgans, J., and H.C. Diener. "Clinical Evidence for Functional Compartmentalization of the Cerebellum" In Cerebellar Functions, edited by J.R. Bloedel and J. Dichgans, 126-47. Berlin-Heidelberg: Springer Verlag, 1984.

Durr, Alexandra. 2010. "Autosomal Dominant Cerebellar Ataxias: Polyglutamine Expansions and Beyond" Lancet Neurology 9(9): 885-94.

Flament, D., and J. Hore. 1986. "Movement and Electromyographic Disorders Associated with Cerebellar Dysmetria" Journal of Neurophysiology 55(6): 1221-33.

Franz, Elizabeth A., Richard B. Ivry, and Laura L. Helmuth. 1996. "Reduced Timing Variability in Patients with Unilateral Cerebellar Lesions during Bimanual Movements" Journal of Cognitive Neuroscience 8: 107-18.

Franz, Elizabeth A., Howard N. Zelaznik, and Anne Smith. 1992. "Evidence of Common Timing Processes in the Control of Manual, Orofacial, and Speech Movements" Journal of Motor Behavior 24(3): 281-7.

Gerwig, Marcus et al. 2003. "Comparison of Eyeblink Conditioning in Patients with Superior and Posterior Inferior Cerebellar Lesions" Brain 126: 71-94.

Gibbon, John. 1977. "Scalar Expectency Theory and Weber's Law in Animal Timing” Psychological Review 84: 279-325.

Gibbon, John, and Russell M. Church. 1990. "Representation of Time" Cognition 37(1-2): 23-54.

Glickstein, Mitchell. 1994. “Cerebellar Agenesis” Brain 117(5): 1209-12. 
Gooch, Cynthia M. et al. 2010. "Interval Timing Disruptions in Subjects with Cerebellar Lesions" Neuropsychologia 48(4): 1022-31.

Gormezano, I. et al. 1962. "Nictitating Membrane: Classical Conditioning and Extinction in the Albino Rabbit" Science 138: 33-4.

Grodd, Wolfgang et al. 2001. "Sensorimotor Mapping of the Human Cerebellum: Fmri Evidence of Somatotopic Organization" Human Brain Mapping 13: 55-73.

Habas, Chrisophe, H. Axelrad, and E.-A. Cabanis. 2004. "The Cerebellar Second Homunculus Remains Silent during Passive Bimanual Movements" NeuroReport 15: 1571-4.

Haken, Hermann, J.A. Scott Kelso, and H. Bunz. 1985. "A Theoretical Model of Phase Transition in Human Hand Movements" Biological Cybernetics 51: 347-56.

Harrington, Deborah L., Kathleen Y. Haaland, and Neal Hermanowicz. 1998. "Temporal Processing in the Basal Ganglia" Neuropsychology 12(1): 3-12.

Harrington, Deborah L. et al. 2004. "Does the Representation of Time Depend on the Cerebellum? Effect of Cerebellar Stroke" Brain 127(3): 1-14.

Herculano-Houzel, Suzana. 2010. "Coordinated Scaling of Cortical and Cerebellar Numbers of Neurons" Frontiers in Neuroanatomy 4: 12.

Hogan, Neville, and Tamar Flash. 1987. "Moving Gracefully: Quantitative Theories of Motor Coordination" Trends in Neuroscience 10: 170-4.

Holmes, Gordon. 1939. "The Cerebellum of Man” Brain 62:1-30.

- 1917. "The Symptoms of Acute Cerebellar Injuries Due to Gunshot Injuries" Brain 40: 461-535.

Hore, Jon, Dagmar Timmann, and Sherry Watts. 2002. "Disorders in Timing and Force of Finger Opening in Overarm Throws Made by Cerebellar Subjects" Annals of the New York Academy of Sciences 978: 1-15.

Hutchinson, Siobhan et al. 2003. "Cerebellar Volume of Musicians" Cerebral Cortex 13(9): 943-9.

Ivry, R., and R.M.C. Spencer. 2004a. "Evaluating the Role of the Cerebellum in Temporal Processing: Beware of the Null Hypothesis" Brain 127(8): E13.

- 2004b. "The Neural Representation of Time" Current Opinion in Neurobiology 14: $225-32$.

Ivry, Richard B. 1996. “The Representation of Temporal Information in Perception and Motor Control" Current Opinion in Neurobiology 6: 851-7.

Ivry, Richard B., and Daniel M. Corcos. "Slicing the Variability Pie: Component Analysis of Coordination and Motor Dysfunction" In Variability and Motor Control, edited by K.M. Newell and D.M. Corcos, 415-47. Urbana, IL: Human Kinetics, 1993.

Ivry, Richard B., and R. Elliot Hazeltine. 1995. "Perception and Production of Temporal Intervals across a Range of Durations: Evidence for a Common Timing Mechanism" Journal of Experimental Psychology: Human Perception and Performance 21(1): $3^{-18 .}$ 
Ivry, Richard B., Steve W Keele, and H.C. Diener. 1988. "Dissociation of the Lateral and

Medial Cerebellum in Movement Timing and Movement Execution" Experimental Brain Research 73: 167-80.

Ivry, Richard B., and Steve W. Keele. 1989. "Timing Functions of the Cerebellum" Journal of Cognitive Neuroscience 1: $136-52$.

Ivry, Richard B., and John E. Schlerf. 2008. "Dedicated and Intrinsic Models of Time

Perception" Trends in Cognitive Sciences 12(7): 273-80.

Ivry, Richard B., Rebecca M.C. Spencer, Howard N. Zelaznik, and Joern Diedrichsen. 2002. "The Cerebellum and Event Timing" Annals of the New York Academy of Sciences 978: 302-17.

Karmarkar, Uma R., and Dean V. Buonomano. 2007. "Timing in the Absence of Clocks: Encoding Time in Neural Network States" Neuron 53(3): 427-38.

Keele, Steve W., and Harold L. Hawkins. 1982. "Explorations of Individual Differences Relevant to High Level Skill" Journal of Motor Behavior 14(1): 3-23.

Keele, Steve W. et al. 1985. "Do Perception and Motor Production Share Common Timing Mechanisms: A Correctional Analysis" Acta Psychologica 6o(2-3): 173-91.

Kelly, Roberta M., and Peter L. Strick. 2003. "Cerebellar Loops with Motor Cortex and Prefrontal Cortex of a Nonhuman Primate" Journal of Neuroscience 23(23): 8432-44.

Kelso, J.A. Scott. 1984. "Phase Transitions and Critical Behavior in Human Bimanual Coordination" Americal Journal of Physiology 15: 1000-4.

Krienen, Fenna M., and Randy L. Buckner. 2009. "Segregated Fronto-Cerebellar Circuits Revealed by Intrinsic Functional Connectivity" Cerebral Cortex 19(10): 2485-97.

Lewis, Penelope A., and R. Chris Miall. 2003. "Brain Activation Patterns during Measurement of Sub- and Supra-second Intervals" Neuropsychologia 41: 1583-92.

Macchi, G., and M. Bentivoglio. "Agenesis or Hypoplasia of Cerebellar Structures" In Handbook of Clinical Neurology, edited by P.J. Vinken and G.W. RBruyn, 367-93. Amsterdam: North-Holland, 1977.

Macdonell, Richard A., Renate M. Kalnins, and Geoffrey A. Donnan. 1987. "Cerebellar Infarction: Natural History, Prognosis, and Pathology" Stroke 18(5): 849-55.

Mangels, Jennifer A., Richard B. Ivry, and Naomi Shimizu. 1998. "Dissociable Contributions of Prefrontal and Neocerebellar Cortex to Time Perception" Cognitive Brain Research 7: 15-39.

Marelli, Cecilia et al. 2011. "Sca15 Due to Large Itprı Deletions in a Cohort of 333 White Families with Dominant Ataxia" Archives of Neurology 68(5): 637-43.

Matell, Matthew S., Warren H. Meck, and Miguel A.L. Nicolelis. 2003. "Interval Timing and the Encoding of Signal Duration by Ensembles of Cortical and Striatal Neurons" Behavioral Neuroscience 117(4): 760-73.

McCormick, David A., and Richard F. Thompson. 1984. "Cerebellum: Essential Involvement in the Classically Conditioned Eyelid Response" Science 223(4633): 296-9. 
Middleton, Frank A., and Peter L. Strick. 2001. "Cerebellar Projections to the Prefrontal Cortex of the Primate" Journal of Neuroscience 21(2): 700-12.

Muzaimi, M.B. et al. 2004. "Population Based Study of Late Onset Cerebellar Ataxia in South East Wales" Journal of Neurology, Neurosurgery, and Psychiatry 75(8): 1129-34.

Nitschke, Matthias F. et al. 1996. "Somatotopic Motor Representations in the Human Anterior Cerebellum: A High-Resolution Functional mri Study" Brain 119: 1023-9.

Nowak, Dennis A., Dagmar Timmann, and Joachim Hermsdorfer. 2007. "Dexterity in Cerebellar Agenesis" Neuropsychologia 45(4): 696-703.

O’Boyle, Donald J., John S. Freeman, and Fredrick W.J. Cody. 1996. "The Accuracy and Precision of Timing of Self-paced, Repetitive Movements in Subjects with Parkinson's Disease" Brain 119: 51-70.

Oakley, David A., and I. Steele Russell. 1972. "Neocortical Lesions and Pavlovian Conditioning" Physiology and Behavior 8(5): 915-26.

Penhune, Virginia B., Robert J. Zatorre, and Alan C. Evans. 1998. "Cerebellar Contributions to Motor Timing: A Pet Study of Auditory and Visual Rhythm Reproduction" Journal of Cognitive Neuroscience 10(6): 752-65.

Perrett, Stephan P., Blenda P. Ruiz, and Michael D. Mauk. 1993. "Cerebellar Cortex Lesions Disrupt Learning-Dependent Timing of Conditioned Eyelid Responses” Journal of Neuroscience 13(4): 1708-18.

Posner, Michael I. Chronometric Explorations of Mind. Hillsdale, NJ:Lawerence Erlbaum Associates, 1978.

Rijntjes, Michel et al. 1999. "Multiple Somatotopic Representations in the Human Cerebellum" NeuroReport 10: 3653-8.

Robertson, Shannon D. et al. 1999. "Correlations for Timing Consistency among Tapping and Drawing Tasks: Evidence against a Single Timing Process for Motor Control" Journal of Experimental Psychology: Human Perception and Performance 25: 1316-30.

Schalling, Elika, Britta Hammarberg, and Lena Hartelius. 2007. "Perceptual and Acoustic Analysis of Speech in Individuals with Spinocerebellar Ataxia (Sca)" Logopedics Phoniatrics Vocology 32(1): 31-46.

Schlerf, John E. et al. 2010. "Evidence of Somatotopy in the Lateral Cerebellar Hemisphere Associated with Complex Actions” Journal of Neurophysiology 103(6): 3330-6.

Schols, Ludger et al. 2004. "Autosomal Dominant Cerebellar Ataxias: Clinical Features, Genetics, and Pathogenesis" Lancet Neurology 3(5): 291-304.

Semjen, Andras, Jeffrey J. Summers, and Daniel Cattaert. 1995. "Hand Coordination in Bimanual Circle Drawing" Journal of Experimental Psychology: Human Perception and Performance 21(5): 1139-57.

Spencer, Rebecca M.C., and Richard B. Ivry. "Cerebellum and Timing" In Handbook of the Cerebellum and Cerebellar Disorders, edited by Mario Manto, Jeremy 
D. Schmahmann, Ferdinando Rossi, Donna L. Gruol and Norkyuki Koibuchi. New York: Springer, 2013.

- 2005. "Comparison of Patients with Parkinson's Disease or Cerebellar Lesions in the Production of Periodic Movements Involving Event-based or Emergent Timing" Brain and Cognition 58: 84-93.

- 2009. "Sequence Learning Is Preserved in Individuals with Cerebellar Degeneration When the Movements are Directly Cued" Journal of Cognitive Neuroscience 21(7): 1302-10.

Spencer, Rebecca M.C., Uma R. Karmarkar, and Richard B. Ivry. 2009. "Evaluating Dedicated and Intrinsic Models of Temporal Encoding by Varying Context" Philosophical Transactions of the Royal Society of London, Series B 364(1525): 1853-63.

Spencer, Rebecca M.C. et al. 2007. "Cerebellar Activation during Discrete and Not Continuous Timed Movements: An Fmri Study" NeuroImage 36: 378-87.

Spencer, Rebecca M.C. et al. 2003. "Disrupted Timing of Discontinuous Movements by Cerebellar Lesions” Science 300: 1437-9.

Spencer, Rebecca M., and Howard N. Zelaznik. 2003. "Weber (Slope) Analyses of Timing Variability in Tapping and Drawing Tasks" Journal of Motor Behavior 35(4): 371-81.

Swanson, Larry W. 1995. "Mapping the Human Brain: Past, Present, and Future" Trends in Neurosciences 18(11): 471-4.

Tesche, Claudia D., and Jari J.T Karhu. 200o. "Anticipatory Cerebellar Responses during Somatosensory Omission in Man" Human Brain Mapping 9: 119-42.

Theoret, Hugo, Jasmine Haque, and Alvaro Pascual-Leone. 20o1. "Increased Variability of Paced Finger Tapping Accuracy Following Repetitive Magnetic Stimulation of the Cerebellum in Humans" Neuroscience Letters 306: 29-32.

Timmann, Dagmar et al. 200o. "Predictive Control of Muscle Responses to Arm Perturbations in Cerebellar Patients" Journal of Neurology, Neurosurgery, and Psychiatry 69(3): 345-52.

Timmann, Dagmar, Sherry Watts, and Jon Hore. 1999. "Failure of Cerebellar Patients to Time Finger Opening Precisely Causes Ball High-Low Inaccuracy in Overarm Throws" Journal of Neurophysiology 2: 103-14.

Trouillas, P. et al. 1997. "International Coopertative Ataxia Rating Scale for Pharmacological Assessment of the Cerebellar Syndrome" Journal of Neurological Sciences 145: 205-11.

Turvey, Michael T. "Preliminaries to a Theory of Action with Reference to Vision" In Perceiving, Acting, and Knowing, edited by Robert Bransford, 211-265. Hillsdale, NJ: Lawerence Erlbaum, 1977.

van de Warrenburg, B.P. et al. 2002. "Spinocerebellar Ataxias in the Netherlands: Prevalence and Age at Onset Variance Analysis" Neurology 58(5): 702-8. 
Velioglu, Sibel K., Kayhan Kuzeyli, and Mehmet Ozmenoglu. 1998. "Cerebellar Agenesis: A Case Report with Clinical and Mr Imaging Findings and a Review of the Literature" European Journal of Neurology 5(5): 503-6.

Vemmos, K.N. et al. 200o. "The Athens Stroke Registry: Results of a Five-year Hospitalbased Study" Cerebrovascular Diseases 10(2): 133-41.

Voogd, Jan, and Mitchell Glickstein. 1998. "The Anatomy of the Cerebellum" Trends in cognitive sciences $2(9)$ : 307-13.

Wing, Alan M., and A. Kristofferson. 1973. "Response Delays and the Timing of Discrete Motor Responses" Perception and Psychophysics 14: 5-12.

Wojtecki, Lars et al. 2011. "Modulation of Human Time Processing by Subthalamic Deep Brain Stimulation" PLoS One 6(9): e24589.

Woodruff-Pak, Diane S., Michelle Papka, and Richard B. Ivry. 1996. "Cerebellar Involvement in Eyeblink Classical Conditioning in Humans" Neuropsychology 10: $443-58$.

$\mathrm{Xu}$, Duo et al. 2006. "Role of the Olivo-Cerebellar System in Timing" Journal of Neuroscience 26(22): 5990-5.

Yamazaki, Tadashi, and Shigeru Tanaka. 2005. "Neural Modeling of an Internal Clock" Neural Computation 17(5): 1032-58.

Zelaznik, Howard N., Rebecca M.C. Spencer, and Richard B. Ivry. 2002. "Dissociation of Explicit and Implicit Timing in Repetitive Tapping and Drawing Movements" Journal of Experimental Psychology: Human Perception and Performance 28: 575-88.

Zelaznik, Howard N. et al. 2005. "Timing Variability in Circle Drawing and Tapping: Probing the Relationship between Event and Emergent Timing" Journal of Motor Behavior 37(5): 395-403. 